\title{
The perception of stereoscopic transparency
}

\author{
ROBIN A. AKERSTROM and JAMES T. TODD \\ Brandeis University, Waltham, Massachusetts
}

\begin{abstract}
The research in the present article was designed to investigate the phenomenon of stereoscopic transparency, in which overlapping surfaces are perceived simultaneously at different depths in the same visual direction. Four experiments are reported that examined observers' abilities to achieve this phenomenon over a wide range of stimulus conditions. The results indicate that (1) the perceptual segregation of overlapping transparent surfaces is significantly more difficult than are comparable judgments for opaque surfaces; (2) the perception of transparency is impaired by increased depth differences between the overlapping surfaces or by increased element density; and (3) the effect is facilitated when the overlapping depth planes are distinguished by color, but not when they are distinguished by element orientation. The theoretical significance of these results is considered within the context of current competitive/cooperative models of stereo matching.
\end{abstract}

One of the most compelling phenomena in visual perception is the sudden appearance of an object's threedimensional form when a random-dot stereogram has been successfully fused. A fundamental theoretical problem highlighted by this phenomenon concerns the matching of elements presented to each eye. A random-dot stereogram is typically composed of thousands of dots, all of which are structurally identical to one another. In order to achieve stereopsis, each dot presented to one eye must be correctly matched with a corresponding dot presented to the other eye. The problem is to distinguish the correct matches from the much larger set of incorrect matches that are mathematically possible.

Numerous algorithms have been proposed in the effort to provide a theoretical solution to this stereo matching problem (e.g., Dev, 1975; Frisby \& Mayhew, 1980; Julesz, 1971; Marr \& Poggio, 1976; Nelson, 1975; Sperling, 1970). Almost all of these algorithms have been based, either implicitly or explicitly, on the fundamental assumption that visible objects in a natural environment are generally composed of smoothly curved opaque surfaces (see Marr \& Poggio, 1976, 1979). There are two important constraints on the detection of correct matches that can be derived from this assumption: (1) a uniqueness constraint, which requires that each element presented to one eye be matched with only one element presented to the other; and (2) a continuity constraint, which requires that elements observed in neighboring regions of space have similar disparities.

One common suggestion for the biological implementation of these constraints involves competitive and cooperative interactions among neighboring neurons, such that cells tuned to similar disparities excite one another,

This research was supported in part by the National Science Foundation (BNS-8420143), the Office of Naval Research, and the Air Force Office of Scientific Research (F49620-87-C-0018). Requests for reprints should be addressed to James T. Todd, Department of Psychology, Brandeis University, Waltham, MA 02254. whereas cells tuned to dissimilar disparities inhibit one another. When the overall pattern of stimulation forms a locally smooth disparity field (i.e., when one is viewing a smoothly curved opaque surface), the competitive and cooperative interactions will dynamically enhance the activity of neurons responding to correct matches while simultaneously suppressing the activity of neurons responding to incorrect matches.

This type of competitive/cooperative neural network can be quite effective for the detection of correct element matches within random-dot stereograms of smoothly curved opaque surfaces, but there are other situations sometimes encountered in nature for which it is not well suited. One such problematic display, which was first described by Sperling (1970), is now known as the doublenail illusion (Krol \& van de Grind, 1980): If two objects are placed one behind the other in the same visual direction (e.g., if two pins are stuck in a pencil pointed at the bridge of the nose), the viewer cannot fuse them simultaneously, even though both objects are monocularly separate in each eye so that there is no loss of information due to occlusion. Using a competitive model such as Sperling's, this double-nail illusion can be explained by the inhibitory interaction between neurons tuned to different disparities in the same visual direction, which produces a form of "rivalry between depth planes."

A similar state of affairs can also arise when one views overlapping transparent surfaces located at different depths. If the surfaces are densely covered with texture, they will produce multiple disparities in every visual direction. If there are inhibitory connections among the neurons that detect these disparities, as suggested above, then the observer's ability to achieve stereopsis with this type of display should be significantly impaired. The theoretical difficulties posed by transparent surfaces for competitive models of stereo matching have been noted previously by several investigators (see, e.g., Frisby, 1980; Prazdny, 1985). For example, in computer simulations of one such model, Marr, Palm, and Poggio (1978) noted 
that "if the input stereograms portray a transparent surface in front of another surface, the algorithm with our parameter values will usually fail to represent the input accurately." It is not at all certain, however, whether similar difficulties are experienced by human observers. Indeed, a number of stereograms that have been published in the literature (see Frisby, 1980; Julesz, 1971; Marr, Palm, \& Poggio, 1978; Prazdny, 1985) demonstrate clearly that the perception of stereoscopic transparency is possible under appropriate circumstances.

One possible model of the stereo matching process that is capable of dealing with overlapping transparent surfaces has recently been suggested by Prazdny (1985). Prazdny's algorithm is cooperative, but not competitivethat is, it is based on the idea that neighboring neurons tuned to similar disparities excite one another, but that there are no interactions whatsoever between cells tuned to dissimilar disparities. The absence of competitive interactions makes it possible to detect elements with similar visual directions on overlapping depth planes without having them interfere with one another.

Although the perception of stereoscopic transparency is potentially of great importance for evaluating alternative theories of stereo matching, there have been no systematic psychophysical investigations of this phenomenon. The present series of experiments was designed, therefore, in an effort to determine the relative difficulty of perceptually segregating overlapping transparent surfaces over a wide range of conditions.

\section{GENERAL METHODS}

\section{Subjects}

Three graduate students from Brandeis University and the two authors participated in each of the experiments to be described below. All had normal or corrected vision and good stereopsis.

\section{Stimulus Generation}

Random-dot stereograms depicting transparent surfaces were produced with a Lex-90 graphics system and displayed on a 19-in. color monitor. To simulate transparent surfaces, two stereo pairs (one on the top portion of the screen and one on the bottom) were generated with uncrossed disparity. Two other stereo pairs with crossed disparity were then superimposed on the uncrossed pairs, so that they overlapped completely. An example of a stereogram generated with this procedure is shown in Figure 1. Upon fusion, four dotted surfaces can be seen, arranged in two overlapping pairs above and below the fixation point.

In the experimental displays, the visible points on the overlapping surfaces were presented as luminous white dots on a black background. Each stereogram's half-field was composed of a 150 $\times 208$ pixel matrix. The overlapping surfaces were displayed at the top and bottom of this matrix as rectangles of $150 \times 100$ pixels, in which $40 \%$ of the pixels in the combined stereogram were illuminated (i.e., the density $d$ on each individual surface was defined by the relation $d=1.0-\sqrt{1.0-0.4}=22.5 \%$ ). These rectangles were separated by a $150 \times 8$ pixel region, which was uniformly black, except for a $4 \times 4$ pixel fixation point at its center. The purpose of this fixation point was to provide a convenient reference for judging the relative depths of the surfaces, and to allow observers to adjust their vergence prior to the onset of each stimulus presentation. The stereograms were viewed through a Keystone
No. 50 stereoscope, using a chinrest to restrict head movements. The visual angle of the entire dot matrix at the focal plane of the stereoscope was $17.7^{\circ}$ in the horizontal direction and $24.4^{\circ}$ in the vertical direction.

\section{Procedure}

When an observer views a random-dot stereogram depicting overlapping transparent surfaces such as the one shown in Figure 1, there are a number of possible phenomenological effects. Under optimal conditions, the relative depths of all four planes will be readily apparent. However, as conditions are made more difficult (e.g., with shorter presentation times, larger disparities, or larger densities), only some of the planes will be perceptually distinct. This effect varies among different observers. For some observers, the relative depths of the two front (crossed) planes will be clearly discerned, but one or both of the back (uncrossed) planes will be perceptually "captured" by the front planes (cf. Ramachandran, 1986; Ramachandran \& Cavanagh, 1985). For other observers, this effect is reversed, such that the front planes are perceptually captured by the back planes. As conditions are made even more difficult, all of the dots will eventually appear to be positioned at the same depth as the fixation point and the display will be perceived as an incoherent pattern of noise. Sometimes an observer may experience all three of these effects in stages with the same display. At first, an overlapping pair of planes may appear as incoherent noise. With continued viewing, one of the planes may "pop out" in depth as a single coherent surface, and finally, the second surface may eventually "pop out"' at a different depth, producing the impression of stereoscopic transparency.

The specific procedure employed in these experiments was designed to provide a reasonably precise evaluation of these various phenomenological effects. Observers were required to make four different responses for each stereogram. Two of these judgments were used to indicate the perceived separability of the surfaces-one for the pattern above the fixation point and one for the pattern below. In each case, if the pattern appeared as two distinct overlapping surfaces at different depths, it was rated as "separate." If, on the other hand, it appeared as a single surface, its position in depth relative to the fixation point was indicated with the response "front," "same," or "behind." The other two judgments were used to designate the perceived depth order of the surfaces. First, the observers indicated which of the two surfaces in front of the fixation point appeared closest in depth with the response of "top," " "bottom," or "I can't tell." Then they made a similar judgment for the two surfaces behind the fixation point.

These judgments provided a clear indication of an observer's perception of any given display. Consider, for example, the stereogram shown in Figure 1. (It can be viewed either convergently or divergently, without affecting the depicted structure.) For the perception of stereoscopic transparency to be achieved, two pairs of overlapping surfaces should be clearly visible, with each pair containing one surface in front of the fixation point and another behind. Note that for the two front surfaces, the one on top is closer in depth, and for the two back surfaces, the one on the bottom is closer. Thus, to indicate a correct perception of stereoscopic transparency, an observer would respond "top, separate; bottom, separate; top closer in front; bottom closer in back." Other variations of these responses reveal different phenomenological effects. For example, a response of "top, separate; bottom, separate; top closer in front; can't tell in back" would indicate that the observer perceived a clear separation of the overlapping depth planes, but that the relative positions of the two surfaces behind the fixation point could not be precisely determined. A response of "top, front; bottom, separate; top closer in front; can't tell in back" would indicate that the back surface on top had been perceptually captured by its overlapping neighbor, and that the relative depths of the two front surfaces were perceived correctly. Finally, responses of "top, 

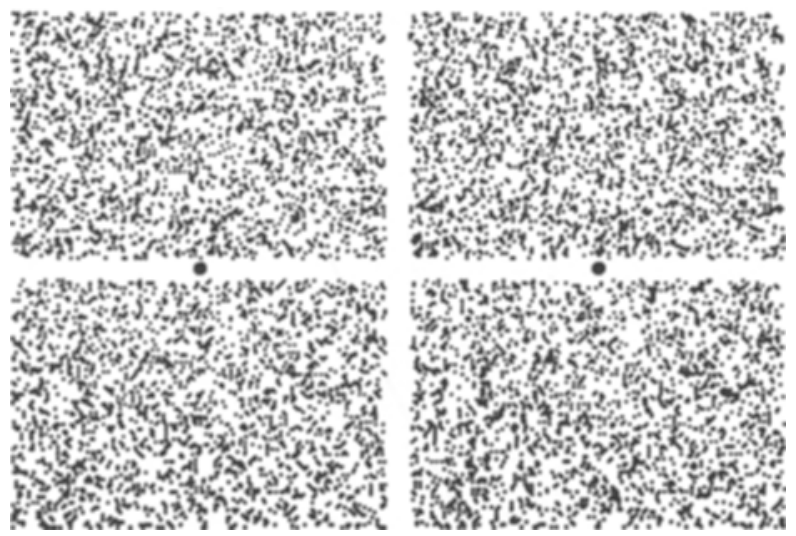

Figure 1. A random-dot stereogram of overlapping transparent surfaces. (In order to minimize the perceptual difficulty of this display, the density of elements has been reduced considerably from that employed in the actual experiments.)

same; bottom, same; can't tell in front; can't tell in back" would indicate the perception of incoherent noise in the plane of the fixation point.

Observers were instructed not to rate a pattern as separate or to attempt to judge the relative depths of the surfaces unless they had a high degree of confidence. Their compliance with this instruction was confirmed after each block of trials by checking the accuracy of their judgments of relative depth.

The difficulty of perceptually segregating overlapping transparent surfaces in a random-dot stereogram can be influenced by a variety of stimulus variables, including the presentation time, the density of the elements in the depicted surfaces, and their relative disparities. An important limiting factor, arising from the spatial resolution of our graphics system, is that disparity could be manipulated in marginal increments of only $7^{\prime}$ of arc. With this scale of values, the difficulty of perceiving stereoscopic transparency increases with disparity. Thus, the easiest display that it was possible to generate depicted surfaces with $7^{\prime}$ and $14^{\prime}$ of both crossed and uncrossed disparity. When we began to pilot the procedure, all 5 observers who participated in the experiment were able to perceive transparency at these minimal disparity values, although they often required an extended viewing period of many seconds before they could perceive the two overlapping surfaces as separately distinct.

In our original experimental design, observers were allowed up to $35 \mathrm{sec}$ to separate the depicted surfaces in each display perceptually. The relative depths of the different surfaces were individually adjusted for each observer in order to obtain his or her upper threshold for disparity in the perception of stereoscopic transparency within a given set of conditions. This procedure worked quite well for 3 of the observers (J.T., S.W., and J.A.), and was adopted to test their performance throughout the entire series of experiments.

This original procedure had to be abandoned for 2 other observers (R.A. and F.R.), however, because their performance gradually improved with practice to the point where they could successfully identify the relative depths of the surfaces even when they were presented with overlapping crossed and uncrossed disparities of several degrees of visual angle. Both observers complained that their successful performance under these conditions produced significant eye strain. Thus, in an effort to reduce the unpleasantness of the task, we adopted an alternative procedure, in which difficulty was manipulated by varying the presentation time. The four surfaces presented in each stereogram were always depicted with $14^{\prime}$ and $28^{\prime}$ of both crossed and uncrossed disparity. The presentation times were individually adjusted for each observer in order to obtain a threshold for exposure duration for the perception of stereoscopic transparency within a given set of conditions.

To summarize briefly, observers were asked to judge the perceived separability and relative depths of overlapping transparent surfaces in random-dot stereograms. Three observers were allowed to view the displays for up to $35 \mathrm{sec}$, and for them the relative disparities of the surfaces were systematically manipulated. For 2 other observers, the presentation times were systematically manipulated for fixed values of relative disparity. These two procedures were used to examine the perception of stereoscopic transparency in a wide variety of experimental conditions. Because many of the observers improved their performance with practice, the specific parameter values for disparity or presentation time were individually adjusted prior to each experiment, in order to avoid ceiling and floor effects. Note, however, that the order of the experiments below has been altered to facilitate our theoretical discussion, which may tend to obscure the observers' overall improvement with practice. The experiments were actually performed as follows: Experiment $3,4,2$, and 1 .

\section{EXPERIMENT 1}

Experiment 1 was designed to compare the relative difficulty of perceptually segregating overlapping transparent surfaces with judgments comparable to those made for opaque surfaces.

\section{Method}

The same response measures as those described in the General Methods section above were employed for both the transparent and opaque conditions: the observers first rated the relative depths of the depicted surfaces, and then judged their perceived separability. Example stereograms depicting transparent and opaque surfaces are shown in Figures 1 and 2, respectively. Note that the same crossed and uncrossed disparities are present in both figures. The only difference is that the depicted surfaces overlap in the transparency condition, and are side by side in the opaque condition. In the actual experimental displays, the combined images of the different surfaces had a uniform statistical dot density of $40 \%$.

The specific parameter values of disparity and exposure time were individually adjusted for each observer in order to isolate their approximate thresholds for perceptually segregating the overlapping surfaces in the transparent condition. Three of the 5 observers (J.T.,
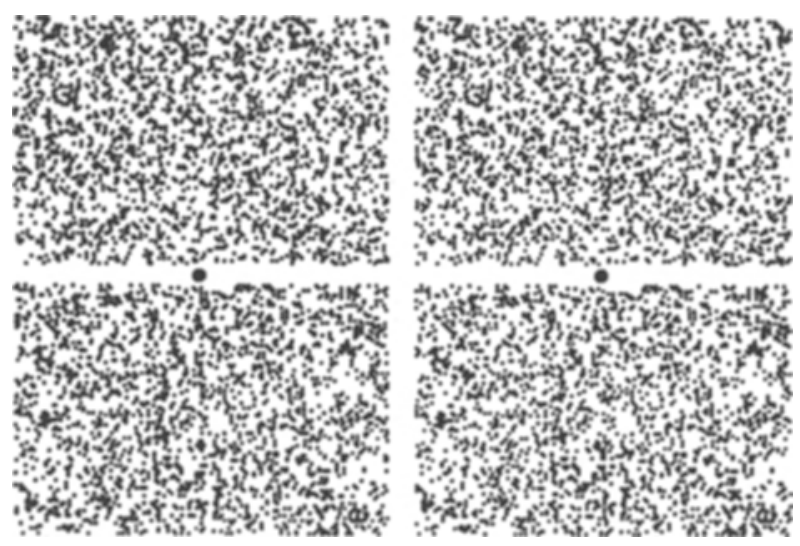

Figure 2. A random-dot stereogram of opaque surfaces similar to those used in Experiment 1. 
S.W., and J.A.) were tested with the disparity manipulation. The crossed/uncrossed disparity pairs used to generate the stimuli were $14^{\prime}$ and $28^{\prime}, 21^{\prime}$ and $35^{\prime}$, and $28^{\prime}$ and $42^{\prime}$ for J.T.; $7^{\prime}$ and $21^{\prime}$, $21^{\prime}$ and $35^{\prime}$, and $35^{\prime}$ and $49^{\prime}$ for S.W.; and $21^{\prime}$ and $35^{\prime}, 35^{\prime}$ and $49^{\prime}$, and $49^{\prime}$ and $63^{\prime}$ for J.A. In each experimental session, the six combinations of display parameters (three disparity pairs $x$ two surface types) were each presented four times in a random order for a total of 24 trials. Four sessions were completed per observer over a period of several days. Over all four sessions, each of the displays was judged 16 times for a total of 32 possible separation judgments per condition.

The other 2 observers (R.A. and F.R.) were tested with the exposure-time manipulation. This time the stereograms were generated with a single crossed/uncrossed disparity pair of $14^{\prime}$ and $28^{\prime}$.
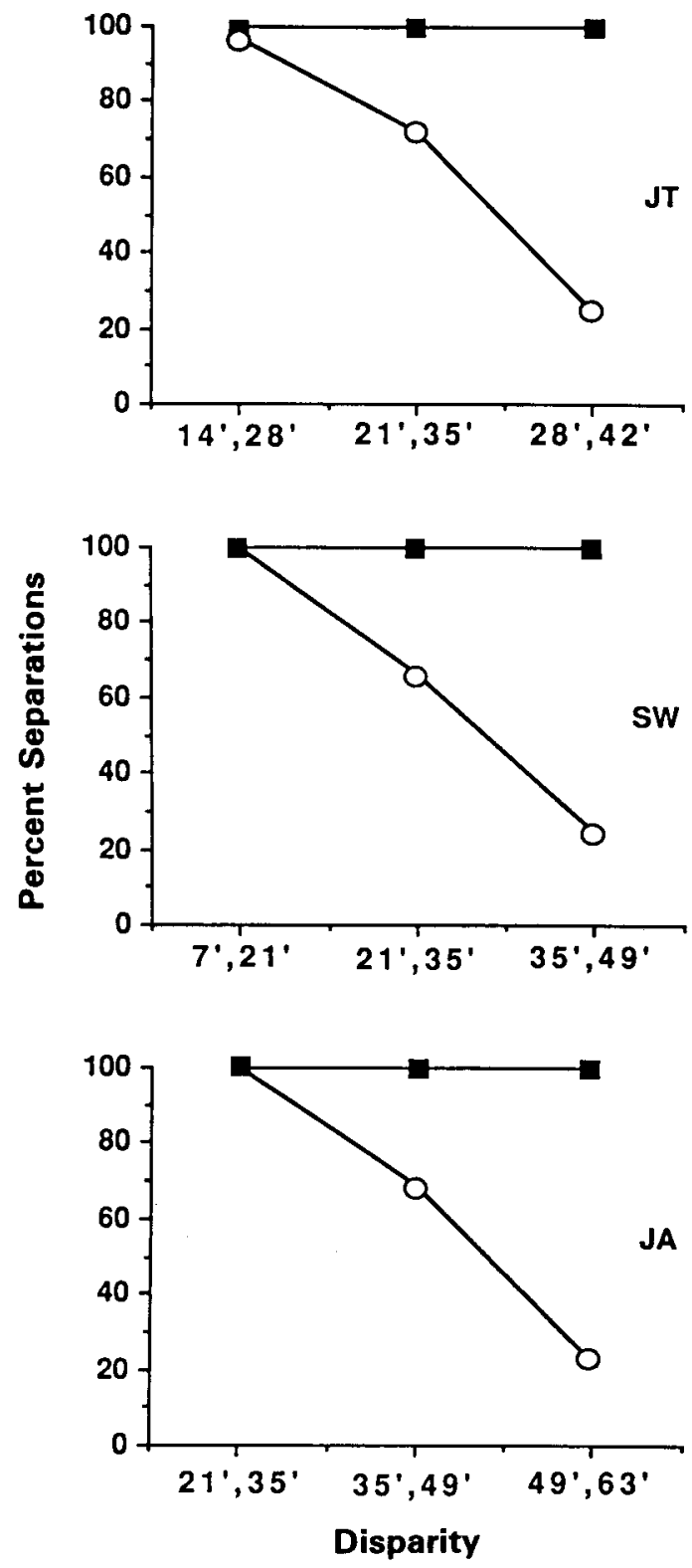

Figure 3. The positive separation judgments obtained in Experiment 1 for 3 observers who were tested with the disparity manipulation. The transparent and opaque conditions are represented by open circles and filled squares, respectively.
The stimulus-exposure durations were 100,300 , and $500 \mathrm{msec}$ for R.A., and 1,500, 2,000, and 2,500 msec for F.R. In each experimental session, the six combinations of display parameters (three exposure durations $\times$ two surface types) were each presented six times in a random order for a total of 36 trials. Three sessions were completed by each of the observers over a period of several days. At the end of the three sessions, each of the displays had been judged 18 times for a total of 36 possible separation judgments per condition.

\section{Results}

Figure 3 shows the percentage of positive separation judgments produced by each combination of display parameters for the 3 observers (J.T., S.W., and J.A.) who were tested with the disparity manipulation. Figure 4 shows the results for the other 2 observers (R.A. and F.R.) who were tested with the exposure-time manipulation. Note that the same general pattern of results was obtained with both experimental procedures. These data show clearly that observers can perceptually segregate overlapping transparent surfaces in random-dot stereograms over a fairly broad range of conditions, but that comparable judgments with opaque surfaces are significantly easier.

The results obtained from the observers' relative depth judgments have not been included in Figures 3 and 4 primarily because they are not directly related to the perception of stereoscopic transparency. It is possible to perceive a clear separation of the overlapping depth planes without having a strong sense of how the different surfaces above and below the fixation point are ordered in depth. Similarly, it is also possible, due to capture effects, to perceive no separation at all, yet still to be able to correctly identify the relative depth order of the surfaces in front of (or behind) the fixation point. In practice, however, the overall patterns of data obtained from the two types of judgments are almost identical. When observers report a clear perceptual segregation of both pairs of overlapping depth planes, they are generally able to identify the relative depth order of the surfaces with $100 \%$ accuracy. Under conditions for which they never perceive separation, they usually respond with "I can't tell" for the depth order judgments.

In addition to the quantitative differences in the observers' perceived difficulty of the displays, some interesting qualitative differences are easily discernible upon careful examination of the two stereograms presented in Figures 1 and 2. Note in the figures that the background regions in between the texture elements appear strikingly different in the two experimental conditions. In the transparent condition, these background regions appear as empty space, in which the individual texture elements are positioned at different depths. For the opaque condition, in contrast, the background regions appear perceptually filled in at the same depth as the surrounding texture, thus creating the impression of a smoothly continuous planar surface.

During their debriefing sessions, most of the observers reported that they often employed specific strategies for directing their attention and eye movements in order to 


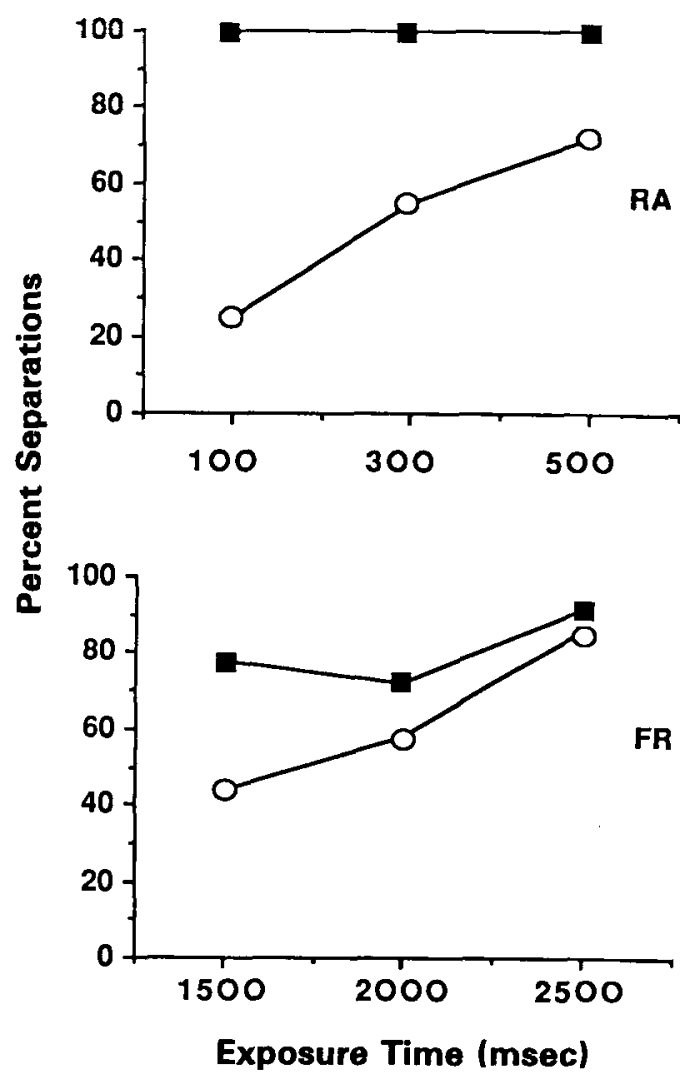

Figure 4. The positive separation judgments obtained in Experiment 1 for 2 observers who were tested with the exposure-time manipulation. The transparent and opaque conditions are represented by open circles and filled squares, respectively.

facilitate a perceptual segregation of the displays, especially in the transparency condition. For this particular experiment, one of the observers (F.R.) noted that her usual strategy for perceptually segregating overlapping transparent surfaces seemed to be disrupted by combining the transparent and opaque conditions within a mixedblock design. This was also confirmed by the data, in that her threshold exposure duration increased dramatically from earlier experiments in which only transparent surfaces were presented (e.g., see Experiments 3 and 4). This effect was not experienced, however, by any of the other observers.

\section{EXPERIMENT 2}

Experiment 2 was designed to examine how the perception of stereoscopic transparency is influenced by variations in texture density.

\section{Method}

The stimuli and procedure here were basically the same as those used in the transparency condition of Experiment 1 . The primary difference was that the statistical dot density of the displays was systematically manipulated from possible values of $15 \%, 25 \%, 35 \%$, and $45 \%$ for the combined monocular views of the overlapping surfaces. Some example stereograms with high- and low-density texture are presented in Figure 5.
As in Experiment 1, the specific parameter values of disparity and exposure time used to generate the displays were individually adjusted for each observer in order to avoid ceiling and floor effects. Three of the observers (J.T., S.W., and J.A.) were given long exposure durations of $35 \mathrm{sec}$, with crossed/uncrossed disparities of $14^{\prime}$ and $28^{\prime}$ for J.T. and $28^{\prime}$ and $42^{\prime}$ for S.W. and J.A. The other two observers (R.A. and F.R.) were given short exposure durations, $300 \mathrm{msec}$ for R.A. and $700 \mathrm{msec}$ for F.R., with crossed/uncrossed disparities of $14^{\prime}$ and $28^{\prime}$.

All of the observers participated in three experimental sessions, in which the four densities were each presented six times in a random order. Thus, over all three sessions, each density was presented 18 times for a total of 36 possible separation judgments per condition.

\section{Results and Discussion}

The percentage of positive separation judgments at each of the four densities is presented in Figure 6 for Observers J.T., S.W., and J.A., and in Figure 7 for Observers R.A. and F.R. As is evident from the figures, the perception of stereoscopic transparency was significantly more difficult with high-density stereograms for 4 of the 5 observers.

To summarize briefly, our results thus far have revealed four basic findings: (1) The perceptual segregation of overlapping transparent surfaces can be achieved within a fairly broad range of stimulus conditions; (2) this segre-
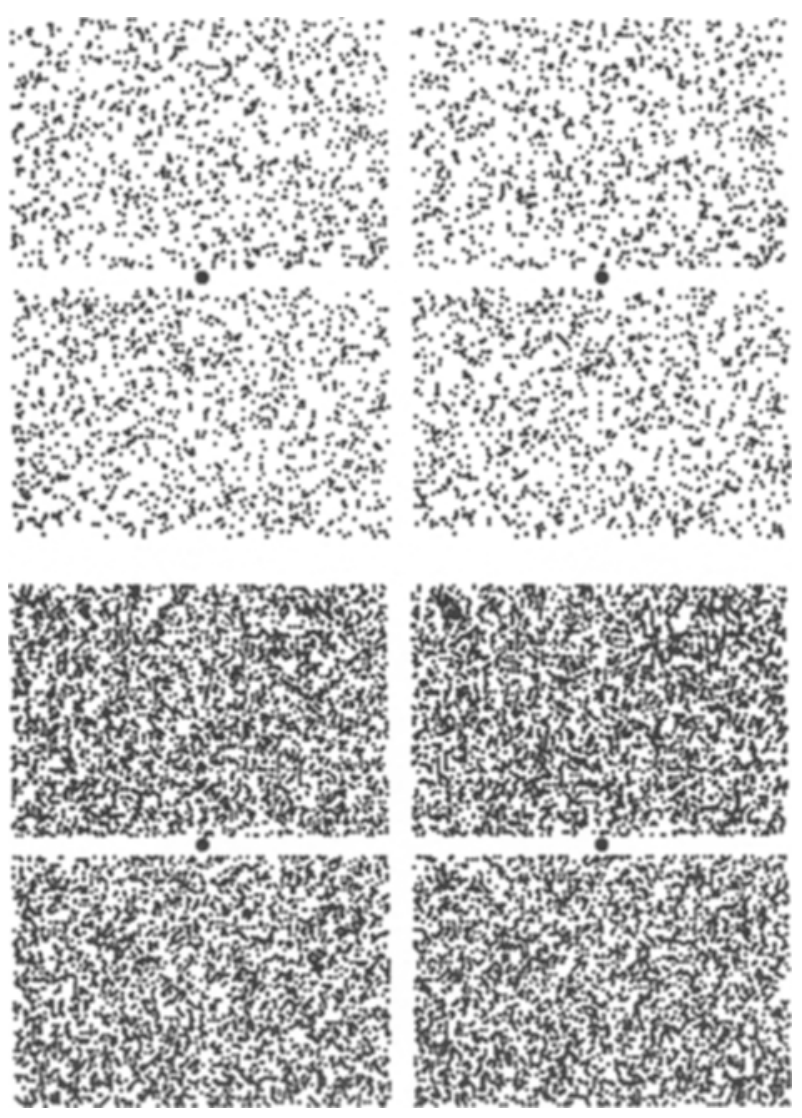

Figure 5. Random-dot stereograms of overlapping transparent surfaces with varying texture density similar to those used in Experiment 2. 
gation is significantly more difficult than is required for comparable displays of opaque surfaces; (3) the difficulty of achieving stereoscopic transparency increases with the depth difference between the overlapping surfaces; and (4) it also increases with the density of matchable features on those surfaces.

Let us now consider some possible theoretical explanations for these findings. One plausible algorithm for solving the problem of the stereo correspondence of transparent surfaces was recently suggested by Prazdny (1985). In Prazdny's algorithm, similar disparity matches in the same local neighborhood facilitate one another's response strength without the aid of inhibitory interactions. The
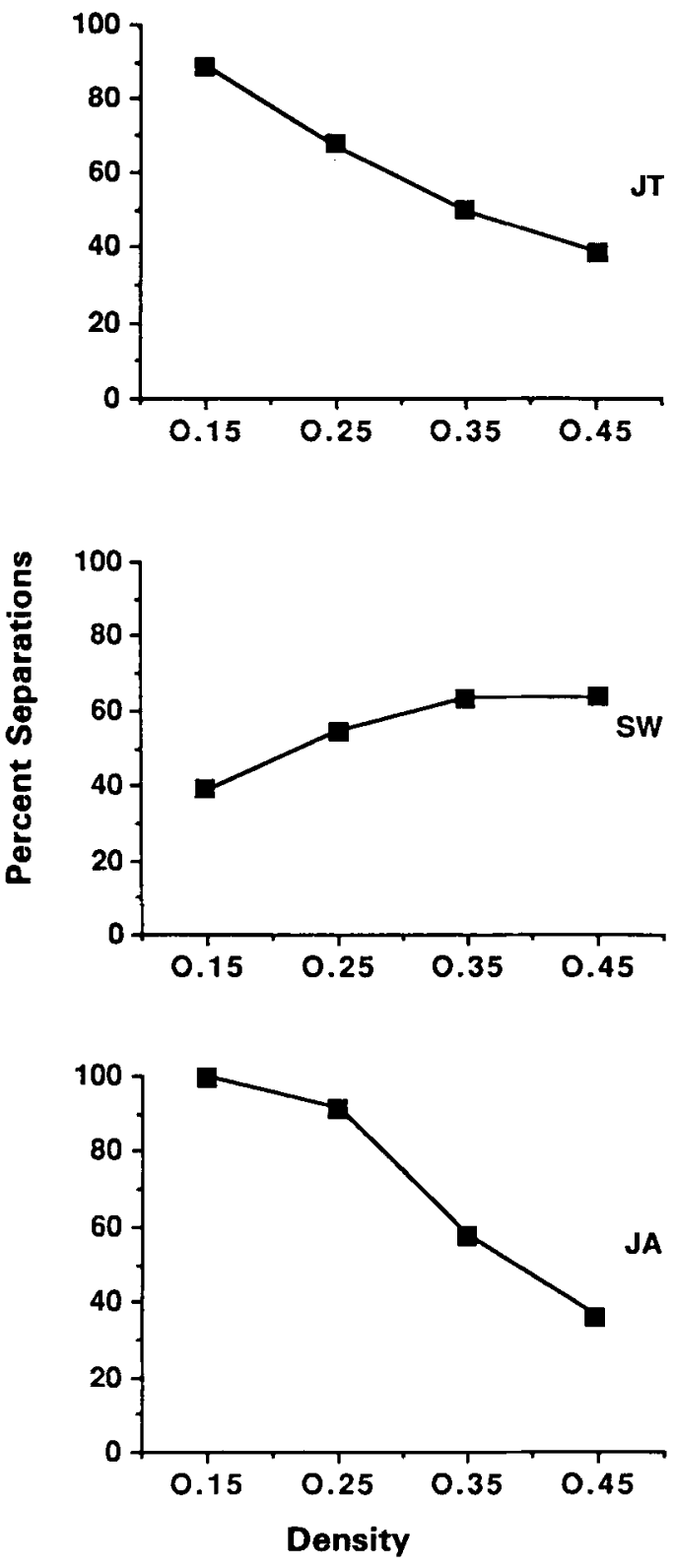

Figure 6. The positive separation judgments obtained in Experiment 2 for 3 observers who were tested with the disparity manipulation.

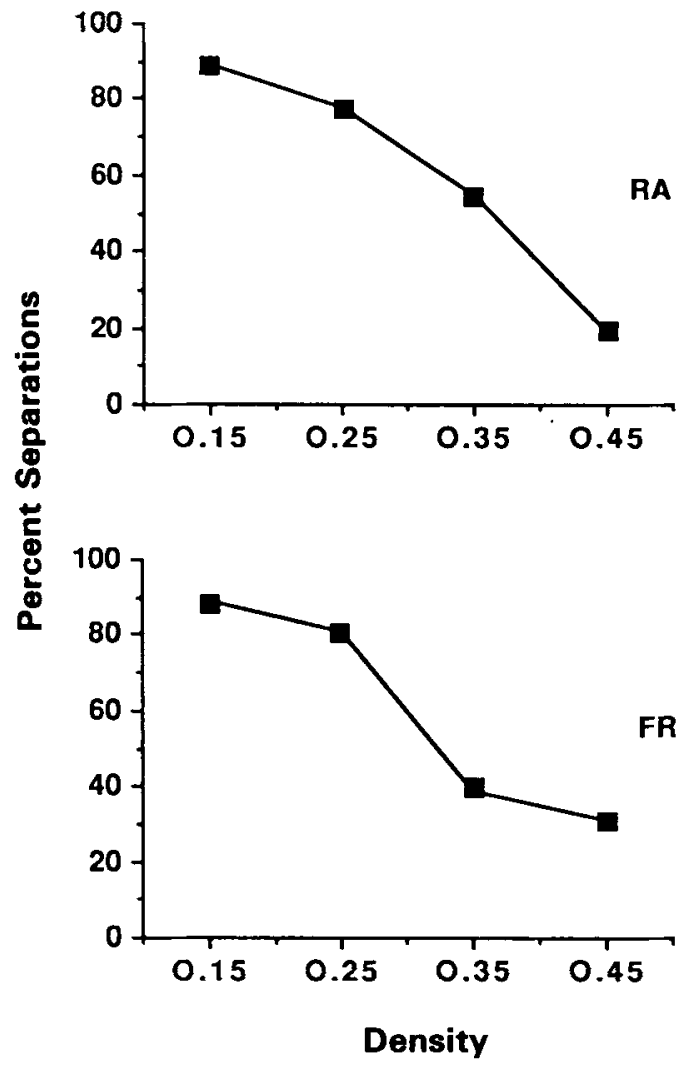

Figure 7. The positive separation judgments obtained in Experiment 2 for 2 observers who were tested with the exposure-time manipulation.

amount of facilitation is inversely proportional to the disparity difference between the neighboring matches and their spatial distance from one another. Among all possible matches for any given feature, the algorithm selects the one with the highest possible response strength. Prazdny tested the performance of this algorithm with a variety of different stimuli, including natural images and random-dot stereograms of both transparent and opaque surfaces. With transparent surfaces the algorithm detected about $75 \%$ of the correct matches, as opposed to nearly $100 \%$ for opaque surfaces. This closely parallels the results obtained in Experiment 1 of the present investigation.

One important reason suggested by Prazdny (personal communication, June 1987) for why his algorithm performs better with opaque surfaces is that the density of correct matches at a given disparity is double that of a comparably dense stereogram of transparent surfaces, in which the correct matches in any local region have two possible disparity values. By reducing the density of similar disparity matches in the neighborhood of interaction, the response strength of those matches would be reduced proportionately, thus making them more difficult to detect. It is important to keep in mind, however, that although this is consistent with previous research using random-dot stereograms of opaque surfaces (e.g., White, 1962), it is fundamentally incompatible with the results 
of Experiment 2. For overlapping transparent surfaces, increasing the density of elements produces an impairment in, rather than a facilitation of, an observer's ability to achieve stereopsis.

One possible explanation for this perplexing effect of density is suggested by the random-dot stereogram of opaque surfaces presented in Figure 2. Note again that in this figure the background regions between the texture elements appear perceptually filled in to form smoothly continuous planar surfaces, and that there are sharply defined subjective contours at the boundaries between regions of different disparity. These observations suggest two general design principles for a biological solution to the stereo matching problem: (1) that locally detected disparity information can propagate into neighboring regions of visual space (cf. Julesz, 1971); and (2) that this propagation is inhibited when two regions of sufficiently different disparity come in contact with one another (cf. Grossberg, 1987a, 1987b; Grossberg \& Mingolla, 1985a, 1985b). According to these principles, the diffusion of locally detected disparity information for a stereoscopically transparent surface (e.g., see Figure 1) would quickly be inhibited by regions of different disparity that are detected from an overlapping surface. This would explain why the background in these displays does not become perceptually filled in. Moreover, as the density is increased, and the regions of different disparity become more tightly packed, their competition would intensify until they could eventually begin to annihilate one another. This would explain why increasing density impairs performance for stereograms of transparent surfaces, even though it enhances performance for stereograms of opaque surfaces.

Although it may appear at first that Prazdny's (1985) analysis would be subverted by the addition of inhibitory interactions, we do not believe that this is necessarily the case, depending on the specific details of how the inhibition is actually performed. Suppose, for example, that excitatory interactions among similar disparity detectors were allowed to occur over a relatively broad region of visual space, but that the inhibitory interactions among dissimilar disparity detectors were restricted to a much narrower spatial range. This type of inhibition would be potentially advantageous for helping to sharpen disparity contours, and would still allow the perception of stereoscopic transparency for overlapping surfaces with reasonably sparse textures.

\section{EXPERIMENT 3}

One important aspect of the phenomenon of stereoscopic transparency is that the individual texture elements are perceptually segregated into multiple groups, each of which corresponds to a different surface in depth. When viewed from this perspective, it is reasonable to speculate that this process of perceptual grouping could be facilitated if the groups were distinguished by other stimulus dimensions in addition to disparity. The remaining two experiments described below were designed to consider some possible implications of this hypothesis. Experiment 3 , in particular, was designed to determine if the perceptual segregation of overlapping transparent surfaces is facilitated when the texture elements on different depth planes can be distinguished on the basis of color.

\section{Method}

As in the previous experiments, the stimuli consisted of randomdot stereograms depicting two pairs of overlapping transparent surfaces. For half of these displays, the four depicted surfaces all contained dots of the same color-either red or green-against a black background. This will be referred to as the single-color condition. For the remaining displays, the overlapping depth planes contained differently colored dots, with red in the front and green in the back, or vice versa. Thus, the color of the dots provided potential information about their grouping in depth. This will be referred to as the multicolor condition.

The relative intensities of the red and green dots were adjusted for each observer so that they would all appear equally bright. The appropriate intensity values were determined prior to the actual experiment using a minimal flicker test. An array of dots was presented, in which the color of each dot oscillated rapidly between red and green. For a fixed intensity of red, the observers adjusted the intensity of green until the perception of flicker was minimized.

It is important to recognize that the stimuli generated using this procedure were fundamentally different from those used in earlier investigations of the possible role of color information in stereopsis (e.g., Gregory, 1979; Lu \& Fender, 1972), in that the texture elements in our displays were all defined by sharp luminance contours. The color information in this case should be considered as a featural quality of the texture elements rather than as their sole defining characteristic (cf. Grossberg, 1987a, 1987b; Grossberg \& Mingolla, 1985a, 1985b).

As in Experiment 1, 3 of the observers (J.T., S.W., and J.A.) were tested with the disparity manipulation, at a fixed exposure duration of $35 \mathrm{sec}$. The disparity pairs were $7^{\prime}$ and $14^{\prime}, 14^{\prime}$ and $21^{\prime}$, and $21^{\prime}$ and $28^{\prime}$ for J.T. and $7^{\prime}$ and $21^{\prime}, 21^{\prime}$ and $35^{\prime}$, and $35^{\prime}$ and $49^{\prime}$ for both S.W. and J.A. During each session, the six combinations of disparity and display type were viewed four times each in a random order. Four sessions were completed by each observer over a period of several days, for a total of 32 possible separation judgments per condition.

Observers R.A. and F.R. were tested with the exposure-time manipulation, using a single crossed/uncrossed disparity pair of $14^{\prime}$ and $28^{\prime}$. The exposure durations were 100,300 , and $500 \mathrm{msec}$ for R.A., and 600,800 and $1,000 \mathrm{msec}$ for F.R. During each session, the six combinations of exposure time and display type were viewed six times each in a random order. Three sessions were completed by each observer, for a total of 36 possible separation judgments per condition.

\section{Results}

The results for the observers (J.T., S.W., and J.A.) who were tested with the disparity manipulation are presented in Figure 8; those for the observers (R.A. and F.R.) who were tested with the exposure-time manipulation are shown in Figure 9. These data show clearly that the perceptual segregation of overlapping transparent surfaces is facilitated when the texture elements on overlapping depth planes can be distinguished on the basis of color. A similar result was also obtained for the relative depth judgments. Over all of the different disparities and presentation times for the multicolor condition, the ob- 
servers identified the correct orders of relative depth in $68 \%$ of the possible judgments, as opposed to only $34 \%$ for the single-color condition. These findings are supported by the observers' phenomenological impressions of the displays. All 5 observers agreed that the multicolor displays were noticeably easier.

One possible explanation for these results is that inhibitory neural interactions produced by the overlapping depth planes are somehow diminished by the differences in chro-
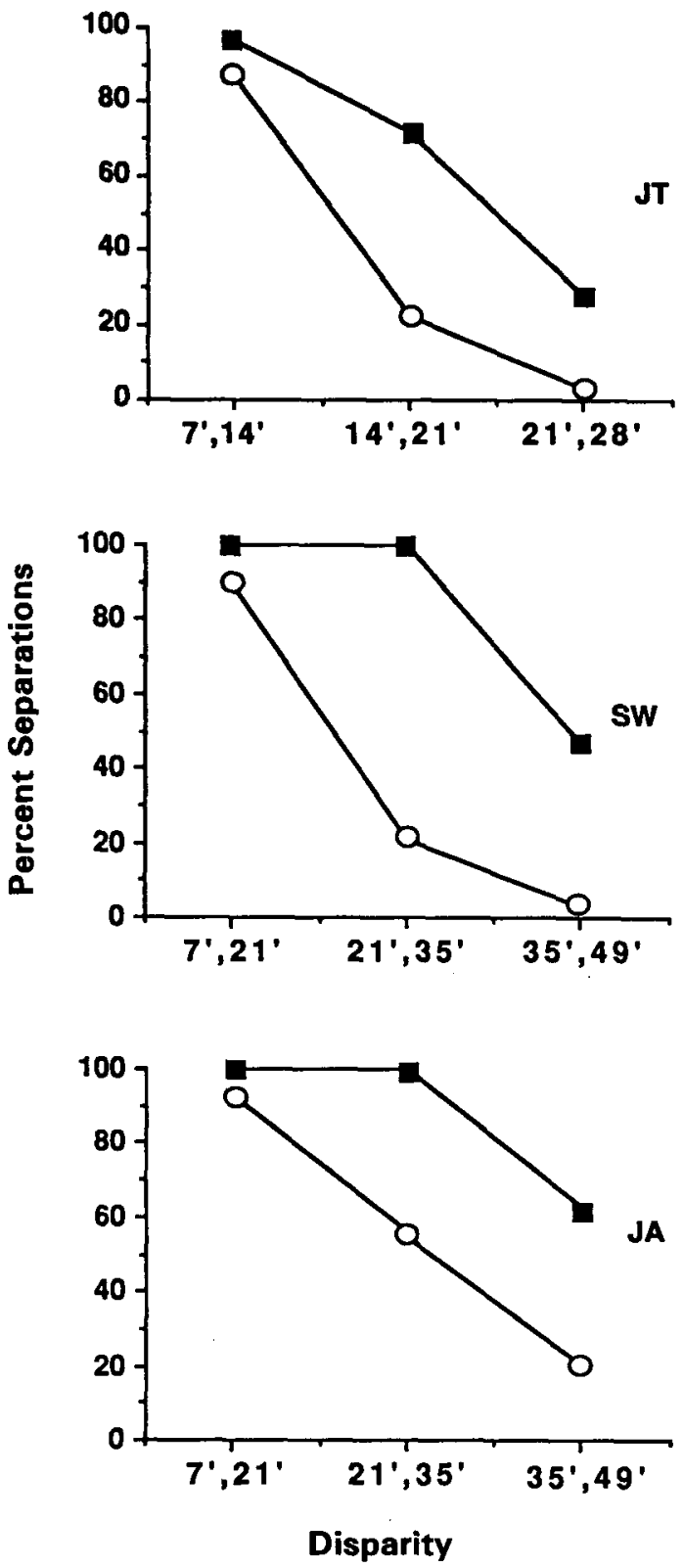

Figure 8. The positive separation judgments obtained in Experiment 3 for 3 observers who were tested with the disparity manipulation. The single-color and multicolor conditions are represented by open circles and filled squares, respectively.

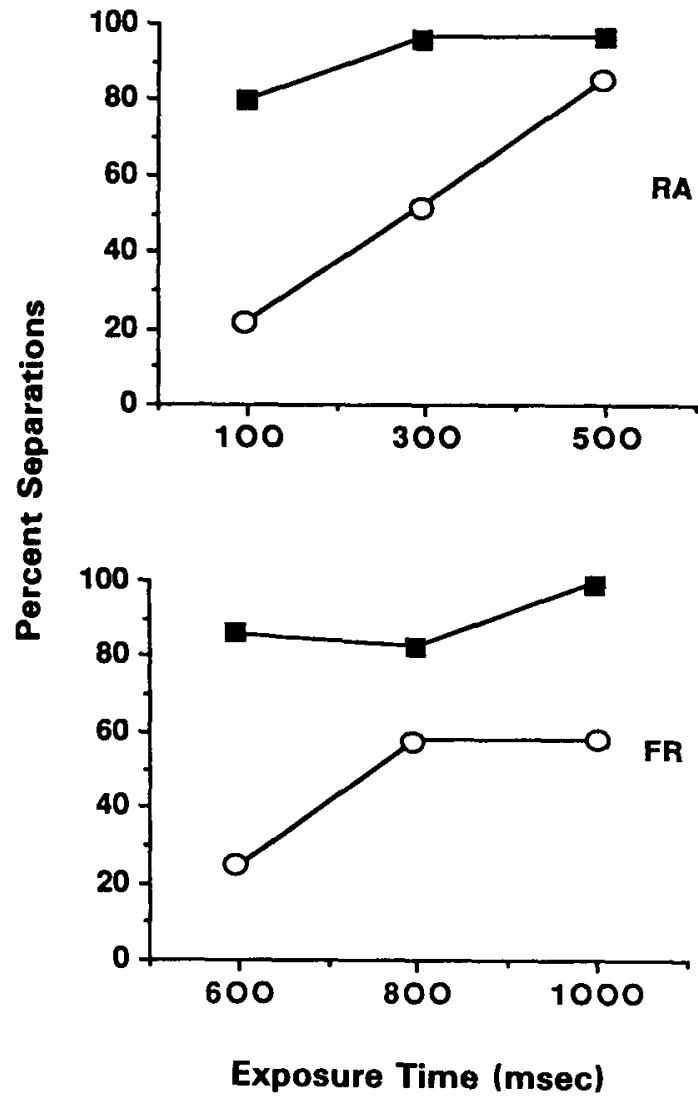

Figure 9. The positive separation judgments obtained in Experiment 3 for 2 observers who were tested with the exposure-time manipulation. The single-color and multicolor conditions are represented by open circles and filled squares, respectively.

matic structure of their respective texture elements. It is interesting in this regard that a similar phenomenon has also been reported by Julesz (1971) and by Julesz and Miller (1975) for variations in spatial frequency. These authors demonstrated that the masking effect of filtered noise on the binocular fusion of random-dot stereograms is greatly attenuated when there is a two-octave separation between the image and noise spectra. They concluded from this result that the processes of human stereopsis receive input from multiple spatial frequency channels that are roughly independent of one another. The independence of these channels has important implications for competitive/cooperative models of stereo matching. By eliminating cross-channel inhibitory interactions, the analysis of incoming information within any given channel cannot be contaminated by noise that is detected at some other spatial frequency.

It is reasonable to speculate that a similar strategy may also be employed in the analysis of color information. Suppose, for example, that there are multiple color channels involved in stereopsis that do not competitively interact during the process of stereo matching. Within such a sys- 
tem, the masking effects of noise would be greatly attenuated when its chromatic structure is sufficiently different from that of the signal for them to be processed along separate channels (e.g., as seems to be the case with spatial frequency). This could also explain why the phenomenon of stereoscopic transparency is easier to achieve when the texture elements on the overlapping depth planes are of different colors.

We have observed other evidence to support this hypothesis by using a slight variation of the present experiment, in which the single-color condition is replaced by one where the overlapping depth planes all contain a $50 \%$ mixture of red and green dots. As with the singlecolor condition, the perception of transparency is significantly more difficult in this case than it is when the colors are grouped on separate depth planes. There are, however, some other interesting phenomenological effects. When the different colored dots are mixed together within each plane, they are not always perceived as a single unit. $\mathrm{Ob}$ servers report that they often experience a perceptual segregation of depths for one color but not for the other, so that the overlapping planes appear to be of unequal density. This observation provides additional evidence that the red and green elements have relatively little influence on one another during the process of stereo matching.

\section{EXPERIMENT 4}

There are many other potential featural qualities in addition to color that could be used to distinguish the texture elements on overlapping transparent surfaces. Thus, in an effort to generalize the effects of color described above, Experiment 4 was designed to determine whether similar effects could be obtained using the property of element orientation.

\section{Method}

As in the previous experiments, the stimuli were designed to simulate two pairs of overlapping transparent surfaces. In this case, however, the displays were composed of oriented line segments instead of dots. Each line segment was 4 pixels long and 1 pixel wide. They were positioned at random in the different depth planes with an appropriate density such that $40 \%$ of the pixels in each stereogram were illuminated. In half of the displays, the line segments on overlapping surfaces could be distinguished from one another by their orientations. That is to say, all of the elements on the front surfaces had identical orientations, which were orthogonal to all of the elements on the back surfaces. This will be referred to as the constant-orientation condition. In the remaining displays, the two possible orientations were mixed together with equal frequency on each surface, so that element orientation would not provide potential information about their grouping in depth. This will be referred to as the mixed-orientation condition. Example stereograms with these two alternative structures are shown in Figure 10.

Observers J.T., S.W., and J.A. were again tested with the disparity manipulation, using a fixed exposure duration of $35 \mathrm{sec}$. The crossed/uncrossed disparity pairs were $14^{\prime}$ and $28^{\prime}, 21^{\prime}$ and $35^{\prime}$, and $28^{\prime}$ and $42^{\prime}$ for J.T.; $21^{\prime}$ and $35^{\prime}, 35^{\prime}$ and $49^{\prime}$, and $49^{\prime}$ and $63^{\prime}$ for S.W.; and $35^{\prime}$ and $49^{\prime}, 49^{\prime}$ and $63^{\prime}$, and $63^{\prime}$ and $77^{\prime}$ for J.A. During each session, the six combinations of disparity and display type were viewed four times each in a random order. Four
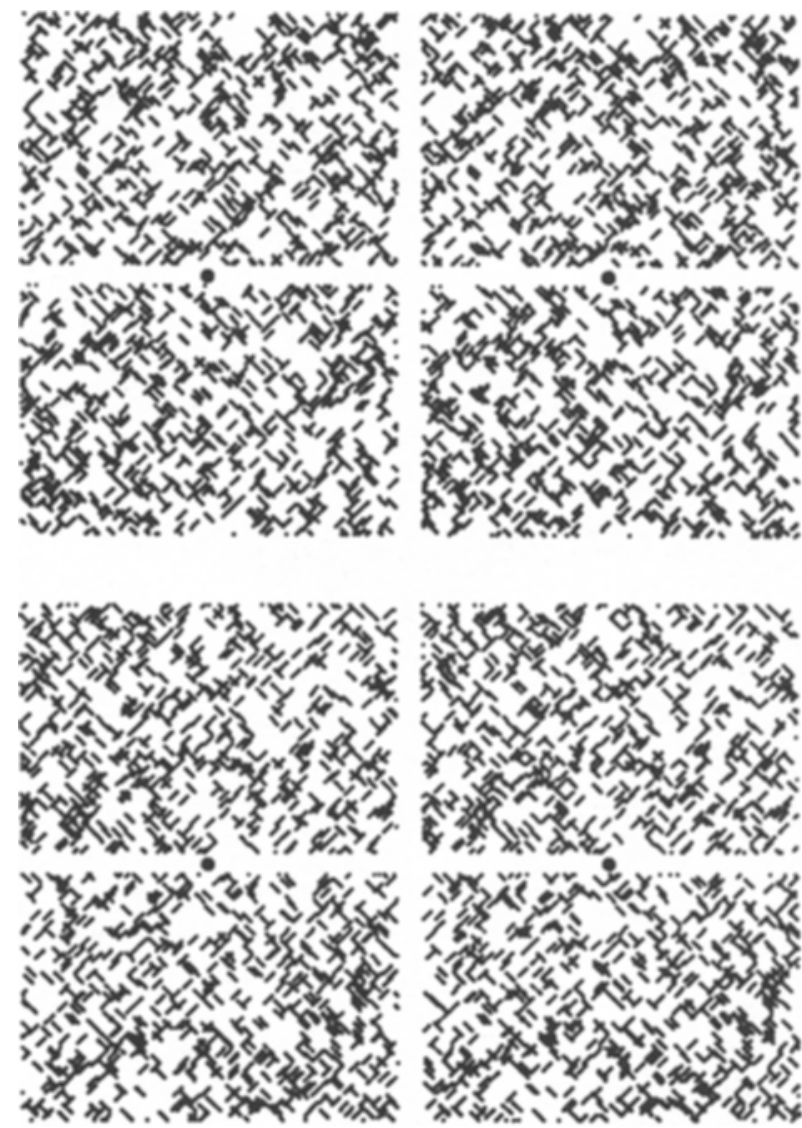

Figure 10. Random-line stereograms of overlapping transparent surfaces similar to those used in Experiment 4. The upper stereogram is from the mixed-orientation condition, and the lower stereogram is from the constant-orientation condition.

sessions were completed by each observer for a total of 32 possible separation judgments per condition.

Observers R.A. and F.R. were tested with the exposure-time manipulation, using a single crossed/uncrossed disparity pair of $14^{\prime}$ and $28^{\prime}$. The exposure durations were 100,300 , and $500 \mathrm{msec}$ for R.A., and 300,500 , and $700 \mathrm{msec}$, for F.R. During each session, the different combinations of exposure duration and display type were viewed six times each, in a random order. Three sessions were completed by each observer, for a total of 36 possible separation judgments per condition.

\section{Results}

The results for the observers (J.T., S.W., and J.A.) who were tested with the disparity manipulation are presented in Figure 11; those for the observers (R.A. and F.R.) who were tested with the exposure-time manipulation are shown in Figure 12. There is little evidence from these data that the perceptual segregation of overlapping transparent surfaces is facilitated when the texture elements on different depth planes can be distinguished by their orientations. Similarly, there was no significant difference between the mixed-orientation and constantorientation conditions in the observers' abilities to correctly identify the relative depth orders of the surfaces. 
These findings are also supported by the observers' phenomenological impressions. All agreed that the grouping of elements by orientation was not a perceptually salient aspect of the displays, and that it had no noticeable effect on the perceived difficulty of achieving stereoscopic transparency.

In comparing these results with the effects of color described in Experiment 3, it is important to keep in mind that when each overlapping depth plane contains a mixture of red and green elements, observers often experience a perceptual segregation of one color but not of the other. No such effects are ever reported for comparable mix-
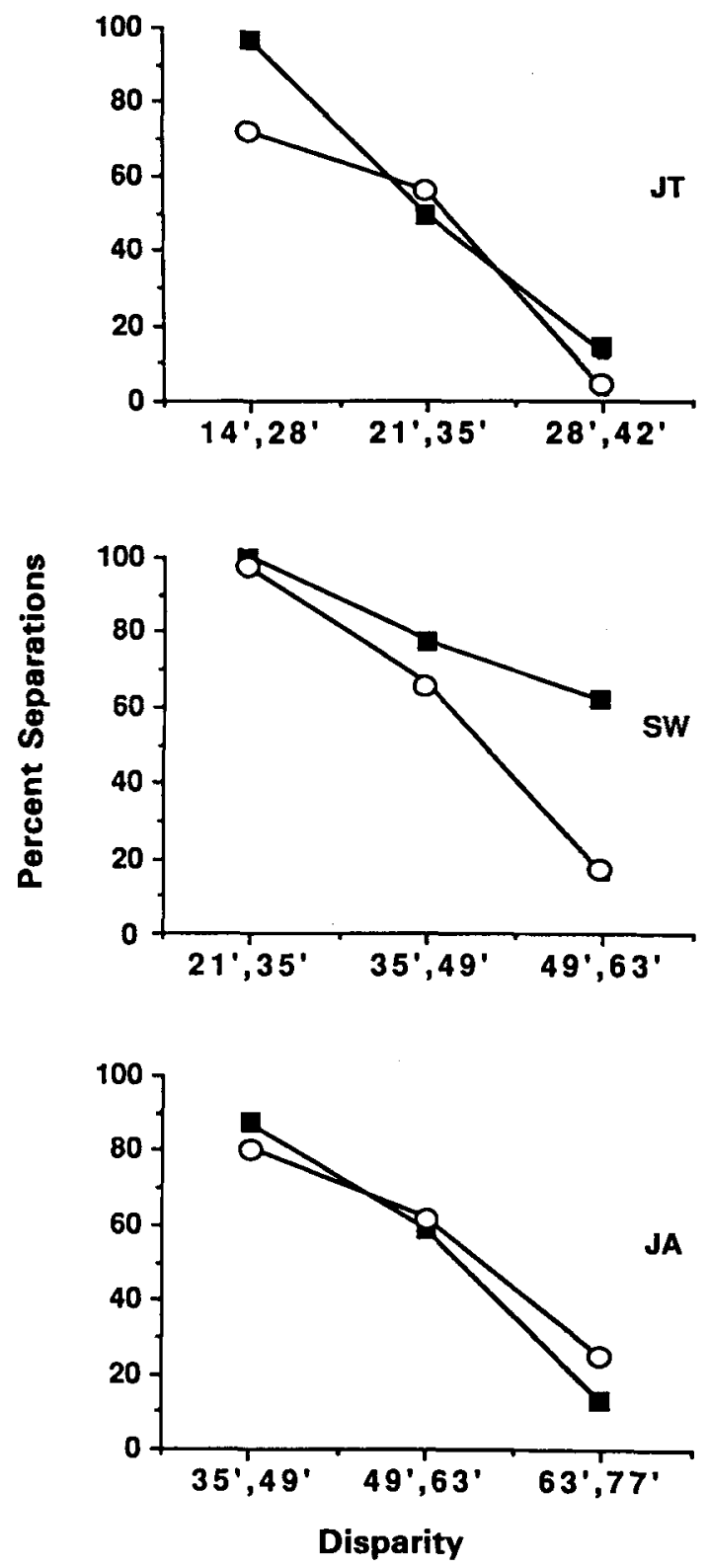

Figure 11. The positive separation judgments obtained in Experiment 4 for 3 observers who were tested with the disparity manipulation. The constant-orientation and mixed-orientation conditions are represented by filled squares and open circles, respectively.

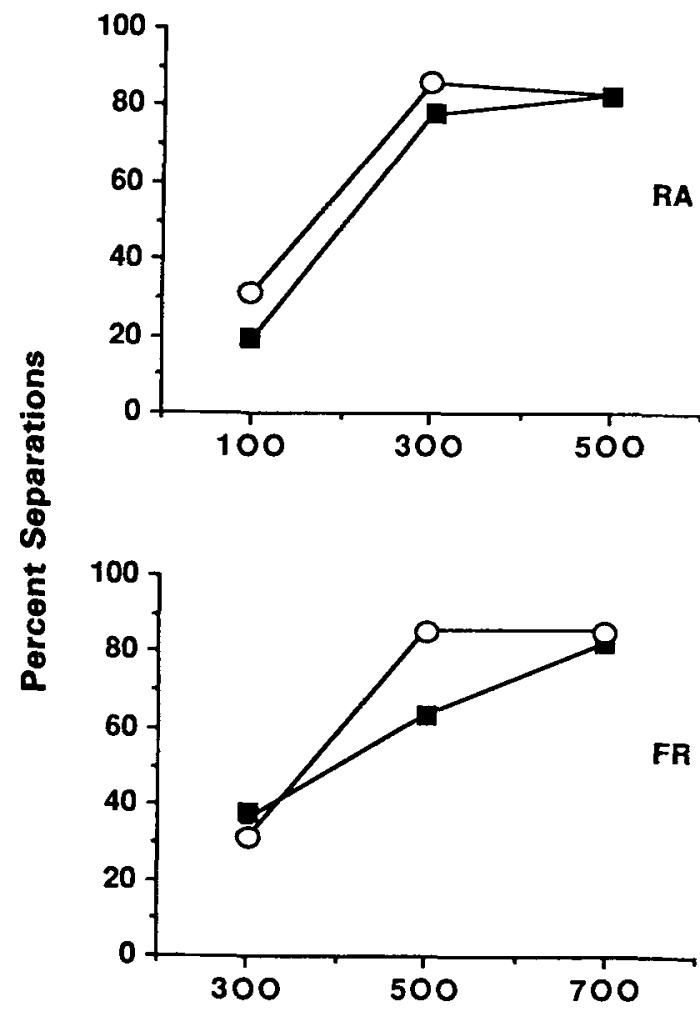

Exposure Time (msec)

Figure 12. The positive separation judgments obtained in Experiment 4 for 2 observers who were tested with the exposure-time manipulation. The constant-orientation and mixed-orientation conditions are represented by filled squares and open circles, respectively.

tures of different element orientations. When the perception of stereoscopic transparency is achieved in the mixedorientation condition of the present experiment, it always occurs for both orientations in an all-or-none manner.

This apparent insensitivity of stereo-matching processes to element orientation has also been suggested in a closely related study by Mayhew and Frisby (1978). These authors examined observers' abilities to achieve stereopsis from oriented random-texture stereograms that were masked by oriented noise. Their results revealed that the specific orientations of the noise elements had no effect whatsoever on observers' perceptions (see also Mayhew \& Frisby, 1979). Taken together, these findings provide strong evidence that the perceptual analysis of randomtexture stereograms does not involve independent orientation channels, as seems to be the case with other stimulus dimensions such as color or spatial frequency.

\section{DISCUSSION}

The research described in the present article has examined the perception of stereoscopic transparency over a broad range of conditions. In Experiments 1 and 2 it 
was demonstrated that the perceptual segregation of overlapping transparent surfaces is significantly more difficult than are comparable judgments for opaque surfaces, and that the perception of transparency is impaired by increasing the depth difference between the overlapping planes or by increasing element density. Experiments 3 and 4 were designed to investigate whether the perceptual segregation of overlapping surfaces in depth could be facilitated if the surfaces were distinguished by other stimulus dimensions in addition to disparity. The results revealed that color differences have a facilitative effect on the perception of stereoscopic transparency but that differences in element orientation do not.

The primary theoretical issue addressed by these experiments concerns the possible neural interactions involved in the process of stereo matching. Many of the models that have been proposed in the literature eliminate false targets using inhibitory connections between neurons tuned to different disparities in the same visual direction (e.g., Dev, 1975; Marr \& Poggio, 1976; Nelson, 1975; Sperling, 1970). An alternate approach developed by Prazdny (1985) is based solely on excitatory interactions among neighboring neurons tuned to similar disparities, with no inhibition whatsoever. The theoretical advantage of this approach is that it allows for the detection of overlapping depth planes without having them interfere with one another.

Although the present results in some ways support Prazdny's analysis, there are several sources of evidence to suggest that the perception of stereoscopic transparency in actual human observers cannot be based on a purely cooperative process, and that overlapping depth planes can indeed produce significant competitive interactions that may impede the detection of correct element matches. One source of evidence is the finding from Experiment 1 that overlapping surfaces are harder to segregate perceptually than nonoverlapping surfaces. There is also the finding from Experiment 2 that the perception of stereoscopic transparency becomes significantly more difficult as the texture elements on overlapping depth planes are packed together in closer and closer proximity. This increased difficulty seems to be the direct result of surface overlap. With random-dot stereograms of opaque surfaces, in contrast, a comparable increase in element density has no significant effect on observers' perceptions (e.g., White, 1962). Finally, the perceptual "filling in" of background regions that typically occurs with random-dot stereograms of opaque surfaces is effectively inhibited when two such surfaces overlap one another (cf. Figures 1 and 2).

All of these results could potentially be explained, we believe, within the general framework of a competitive/ cooperative model of stereo matching. One possibility is that neurons tuned to similar disparities cooperatively interact over relatively large neighborhoods of visual space, whereas competitive interactions among neurons tuned to dissimilar disparities are restricted to much smaller neighborhoods. This type of inhibition would be potentially advantageous for helping to sharpen disparity contours, yet it would still allow for the perception of stereoscopic transparency for overlapping surfaces with reasonably sparse textures.

There are other aspects of our results, however, which strongly suggest that neural interactions based solely on position and disparity cannot tell the whole story. For example, the long presentation times that are required by most observers to achieve the impression of stereoscopic transparency is a compelling source of evidence that vergence eye movements may play a critical role in this phenomenon. Observers typically report that it often requires considerable "effort" for the overlapping surfaces to pop out in depth. Their specific strategies for directing attention and eye movements seem to be particularly important in this regard. Indeed, the effectiveness of these strategies may well be a primary determinant of the large individual differences and practice effects that were observed throughout the course of the investigation.

Another source of evidence that there are additional stimulus properties besides position and disparity that can influence the perception of stereoscopic transparency is provided by Experiment 3 . This experiment demonstrated that the ability of observers to segregate overlapping transparent surfaces perceptually can sometimes be facilitated when the individual depth planes are appropriately labeled with other sources of information, such as colcr. One possible explanation of this effect is that monocularly distinguishable stimulus properties may aid in the control of vergence eye movements. There are two important aspects of the data, however, which suggest that this hypothesis cannot stand on its own. First, although the minimum initiation time of a vergence eye movement is approximately $160 \mathrm{msec}$, one of our observers (R.A.) exhibited facilitation effects with stimulus presentations of only $100 \mathrm{msec}$. Second, given an eye-movement hypothesis, it makes little sense that surfaces distinguished by color should be easier to segregate than those distinguished by element orientation.

Because of these complicating factors, it is probably best to be circumspect about proposing specific models for how observers are able to achieve the perception of stereoscopic transparency. Our results indicate that this ability may involve a complex pattern of competitive and cooperative interactions among a wide variety of stimulus variables, with potentially important contributions from the oculomotor control system. A precise model of how these various factors are organized will have to await future research.

\section{REFERENCES}

Dev, P. (1975). Perception of depth surfaces in random dot stereograms: A neural model. International Journal of Man-Machine Studies, 7 , 511-528.

FrISBY, J. (1980). Seeing: Illusion, brain and mind. New York: Oxford University Press.

FrisBY, J., \& MAYHEW, J. E. W. (1980). Spatial frequency tuned channels: Implications for structure and function from psychophysical and computational studies of stereopsis. Philosophical Transactions of the Royal Society of London, Series B, 290, 95-116. 
Gregory, R. L. (1979). Stereo vision and isoluminance. Proceedings of the Royal Society of London, Series B, 204, 467-476.

Grossberg, S. (1987a). Cortical dynamics of three-dimensional form, color, and brightness perception: I. Monocular theory. Perception \& Psychophysics, 41, 87-116.

GrossBerg, S. (1987b). Cortical dynamics of three-dimensional form, color, and brightness perception: II. Binocular theory. Perception \& Psychophysics, 41, 117-158.

Grossberg, S., \& Mingolla, E. (1985a). Neural dynamics of form perception: Boundary completion, illusory figures, and neon color spreading. Psychological Review, 92, 173-211.

Grossberg, S., \& Mingolla, E. (1985b). Neural dynamics of perceptual grouping: Texures, boundaries, and emergent segmentations. Perception \& Psychophysics, 38, 141-171.

Julesz, B. (1971). Foundations of Cyclopean perception. Chicago: University of Chicago Press.

JULESz, B., Miller, J. E. (1975). Independent spatial frequency tuned channels in binocular fusion and rivalry. Perception, 4, 125-143.

KROL, J. D., \& VAN DE GRIND, W. A. (1980). The double nail illusion: Experiments on binocular vision with nails, needles, and pins. Perception, 9, 651-669.

Lu, C. Fender, D. (1972). The interaction of color and luminance in stereoscopic vision. Investigative Ophthalmology, 11, 482-490.

MARR, D., PAlm, G., \& Poggio, T. (1978). Analysis of a cooperative stereo algorithm. Biological Cybernetics, 28, 223-239.

MARR, D., PoGgio, T. (1976). Cooperative computation of stereo disparity. Science, 104, 283-287.
MARr, D., PoGgio, T. (1979). A computational theory of human stereo vision. Proceedings of the Royal Society of London, Series B, 204, 301-328.

MAYhew, J. E. W., \&RISBY, J. P. (1978). Stereopsis masking in humans is not orientationally tuned. Perception, 7, 431-436.

MAYHEW, J. E. W., \& FrISgY, J. P. (1979). Surfaces with steep variations in depth pose difficulties for orientationally tuned disparity filters. Perception, 8, 691-698.

Nesson, J. I. (1975). Globality and stereoscopic fusion in binocular vision. Journal of Theoretical Biology, 49, 1-88.

Prazdny, K. (1985). Detection of binocular disparities. Biological Cybernetics, 52, 93-99.

RAmachandRan, V. S. (1986). Capture of stereopsis and apparent motion by illusory contours. Perception \& Psychophysics, 39, 361-373.

Ramachandran, V. S., Cavanagh, P. (1985). Subjective contours capture stereopsis. Nature, 317, 527-530.

SPERLING, G. (1970). Binocular vision: A physical and neural theory. American Journal of Psychology, 83, 461-534.

WHITE, B. W. (1962). Stimulus conditions affecting a recently discovered stereoscopic effect. American Journal of Psychology, 75 411-420.

(Manuscript received November 2, 1987; revision accepted May 11,1988 .) 